\title{
La eficiencia terminal del Centro de Investigación e Innovación Tecnológica
}

\section{Completion Rate at the Center for Research and Technological Innovation}

José de Jesús Peinado Camacho (*) jpeinadoc@ipn.mx

David Jaramillo Vigueras ${ }^{*}$ ) djaramillo@ipn.mx

(*) Instituto Politécnico Nacional

(Recibido: 25 de enero de 2017; Aceptado para su publicación: 17 de marzo de 2017)

Cómo citar: Peinado, J. y Jaramillo, D. (2018). La eficiencia terminal del Centro de Investigación e Innovación Tecnológica. Revista Electrónica de Investigación Educativa, 20(3), 126-134. Recuperado de https://doi.org/10.24320/redie.2018.20.3.1797

\section{Resumen}

El objetivo de este trabajo es exponer indicadores de la eficiencia terminal del Centro de Investigación e Innovación Tecnológica del Instituto Politécnico Nacional (México). La metodología que se utilizó fue cuantitativa, se generó un estudio estadístico de la eficiencia terminal de los alumnos y de los profesores con el propósito de establecer la relación entre estas dos variables. Los resultados de la investigación evidencian logros en torno a la eficiencia terminal y revelan áreas de oportunidad para orientar estrategias que favorezcan una mejor selección de los aspirantes.

Palabras clave: Eficiencia terminal, posgrado, estudiantes de posgrado, tutores.

\section{Abstract}

The objective of this study is to present indicators of the completion rate at the Center for Research and Technological Innovation (Mexico). The methodology used was quantitative and a statistical study of students' and teachers' completion rates was generated with the aim of establishing a relationship between the two variables. The findings show an improvement in completion rates and reveal areas of opportunity where strategies may be directed to support the selection of applicants.

Keywords: Terminal efficiency, postgraduate courses, postgraduates, tutors.

\section{Introducción}

En términos generales la eficiencia es el nivel óptimo de funcionalidad y congruencia para concretar las metas con calidad (Hernández, Vargas, Almuiñas y García, 2015). La principal función de una institución de educación superior es la docencia y, por tanto, su eficiencia depende de la proporción de alumnos que logran egresar o titularse, respecto a aquellos que ingresaron, a este indicador se le llama eficiencia terminal (López, Albíter y Ramírez, 2008). 
La eficiencia se asocia necesariamente a las condiciones y los recursos disponibles con que se busca ser eficaz. Por eficaz se entiende la medida en que se cumplen los objetivos y se alcanzan las metas. De este modo, se es eficiente en la medida que se aprovechan los recursos disponibles (tiempo, talento, dinero). Así, la eficiencia es la resultante de una relación entre los objetivos alcanzados y las condiciones en que se trabaja. Por lo que los indicadores relacionados con estos dos criterios tienen que ver con la medida en que se logran las metas y las circunstancias en que esto ocurre, expresándose en términos relativos o proporcionales. Algunos ejemplos que pueden usarse son: la proporción de alumnos en el programa que concluye satisfactoriamente los estudios; la relación entre los objetivos de aprendizaje que contiene el currículo y los efectivamente alcanzados por cada alumno; el tiempo promedio que demanda concluir el programa; la eficiencia terminal; la proporción entre investigadores y proyectos vigentes; la tasa de artículos, libros o reportes por investigador o por proyecto (Cardoso y Cerecedo, 2011).

Algunas universidades utilizan dos indicadores vinculados con la eficiencia académica, desde el punto de vista cuantitativo: la eficiencia académica terminal, que es el número de estudiantes que se gradúan en un ciclo completo; y la eficiencia académica vertical, que es la cantidad de estudiantes aprobados del total de la matricula inicial (Hernández et al., 2015).

Por otra parte Álvarez, Gómez y Morfín (2012), en su estudio sobre el efecto que tiene la beca que otorga el Conacyt para estudios de posgrado sobre los índices de eficiencia terminal de posgrado en México, analizan específicamente la Maestría en Tecnologías para el Aprendizaje del Centro Universitario de la Costa (Universidad de Guadalajara). Su estudio sugiere que no existe relación de la beca de Conacyt con la graduación de estudiantes y, por lo tanto, con la eficiencia terminal del posgrado. Explican que los factores que inciden de manera determinante en la graduación deben buscarse en otros aspectos administrativos o académicos de los posgrados.

Sin embargo, podría existir un efecto inverso de las becas Conacyt sobre la eficiencia terminal. Por ejemplo, el Posgrado de Estudios Latinoamericanos de la Universidad Nacional Autónoma de México (UNAM) redujo al 50\% el número de seleccionados a nuevo ingreso. La coordinación del programa de posgrado explicó que el menor número de jóvenes aceptados obedece a que los comités elevaron los criterios para el ingreso, ya que este posgrado está en riesgo de perder el registro ante Conacyt debido a su baja eficiencia terminal. El promedio de titulación en tiempo y forma es de 16\%, cuando el mínimo establecido por Conacyt es de 40\% (Olivares, 2016).

En el caso del Instituto Politécnico Nacional (IPN) se han dispuesto herramientas para la gestión del posgrado, algunos ejemplos son: la propuesta de un modelo de gestión del conocimiento administrativo para la División de Apoyo al Posgrado del IPN, unidad administrativa responsable de la gestión de los servicios escolares de posgrado del Instituto (Bustos, Cerecedo y García, 2016). El tablero de mando integral como herramienta de planeación estratégica (Peinado, Cerecedo y Jaramillo, 2016), con el cual se pueden implementar estrategias clave para monitorear su desempeño (Fadhl y Sofian, 2015). Otra propuesta es un modelo de gestión del capital intelectual (Peinado, 2014), éste plantea el aprovechamiento de intangibles para promover la productividad científica en los centros de investigación del IPN. Adicionalmente, Álvarez, Chávez, Montiel, Topete y Maldonado (2015) indican que el nivel posgrado en el IPN requiere de una administración descentralizada por áreas de conocimiento.

\subsection{Antecedentes}

El Centro de Investigación e Innovación Tecnológica (CIITEC) fue creado el 24 de julio de 1997. Desde entonces ha desarrollado proyectos de investigación aplicada y desarrollo tecnológico enfocados a la solución de problemas técnicos del sector productivo (Peinado, 2009). El CIITEC surge del Centro de Productividad de la Escuela Superior de Ingeniería Mecánica y Eléctrica, Unidad Azcapotzalco, y se constituyó como una fuente generadora de recursos económicos (Bonilla y Peinado, 2014).

El programa de Maestría en Tecnología Avanzada (MTA) del CIITEC está alineado con el Reglamento de Estudios de Posgrado del IPN, que define los criterios de movilidad y excelencia académica (Bonilla y Peinado, 2014). Este programa conlleva un fuerte componente en los métodos científico y de la 
innovación tecnológica, teniendo por objeto formar personal que participe en el desarrollo de la ciencia y la tecnología. Su plan de estudios fomenta la movilidad y excelencia académica. El programa de Doctorado en Tecnología Avanzada (DTA) del CIITEC, está alineado también con el Reglamento de Estudios de Posgrado del IPN y con las políticas establecidas por la Secretaría de Investigación y Posgrado (sIP). Este programa tiene por objeto formar personal capaz de participar en el desarrollo de la ciencia y la tecnología, asociados a las necesidades de la actividad productiva. La estructura curricular del DTA se basa en un plan semestral que contempla un total de ocho semestres. El plan de estudios es personalizado y enfocado a la realización de actividades de investigación (CIITEC, 2014).

Los dos programas de posgrado del CIITEC están reconocidos por el Programa Nacional de Posgrados de Calidad (PNPC) del Conacyt desde el año 2008. En el 2014 el programa de MTA del CIITEC fue evaluado y promovido al nivel consolidado. El siguiente paso en la MTA del CIITEC es alcanzar el nivel de Competencia Internacional. Después, en 2015, el programa de DTA fue ratificado con nivel en Desarrollo (Conacyt, 2016). En este contexto, la oferta educativa de posgrado del IPN se compone por 147 programas de posgrado, 92 de estos programas están acreditados en el Conacyt, y 8 son programas de Competencia Internacional (IPN, 2016).

Con relación a la eficiencia terminal, la sIP elaboró una propuesta de indicadores de desempeño de investigación y posgrado. La información fue recabada de 19 centros de investigación y examinó 11 indicadores de desempeño, entre los cuales se encuentran: la atención a la demanda de formación académica con calidad, la generación de recursos humanos especializados y la tasa de graduación por cohorte generacional (SIP, 2015).

El indicador de atención a la demanda de formación académica con calidad se obtuvo al multiplicar el número de alumnos aceptados anualmente en el posgrado por 100 , el resultado fue dividido entre el total de alumnos aspirantes al posgrado en el año correspondiente. En este apartado el clitec se ubicó en el quinto sitio con 0.69 (SIP, 2015).

Referente al indicador de generación de recursos humanos especializados, el cual se obtuvo sumando el número de alumnos graduados en programas de especialidad, más el número de alumnos graduados en programas de maestría, más el número de alumnos graduados en programas de doctorado; el resultado fue dividido entre el número de alumnos inscritos. Para reforzar este indicador los programas de posgrado deben estar inscritos en el PNPC. El CIITEC se situó en tercer lugar con 1.33 (SIP, 2015).

Y por último, el indicador de tasa de graduación por cohorte generacional se obtuvo al dividir el número de alumnos que obtuvieron grado académico de una generación (dentro de la duración manifestada por el programa) entre el número de alumnos inscritos en la generación considerada. El CIITEC se colocó en séptimo lugar con 0.65 (SIP, 2015).

\section{Método}

La pregunta principal de esta investigación fue: ¿Cuáles son los factores que afectan directamente la eficiencia terminal en el posgrado? Para dar respuesta a esta interrogante se efectuó un estudio no experimental basado en variables que ya ocurrieron sin la intervención directa del investigador. La metodología que se utilizó fue cuantitativa, se generó un análisis estadístico de la eficiencia terminal de los alumnos (inscritos, activos, graduados y bajas) y de los profesores (directores de tesis) de los semestres A2005 al semestre A2016, con la finalidad de establecer si existe una relación entre estas dos variables. La estrategia metodológica que se utilizó fue el estudio de caso, que examina y analiza a profundidad la interacción de los factores que producen cambios o crecimiento (Ortiz, 2011; Tamayo, 2009), y se utiliza también para contribuir al conocimiento de individuos, grupos, fenómenos organizacionales, sociales, políticos y otros relacionados (Yin, 2014). 


\section{Análisis}

Previamente se efectuó la búsqueda de información histórica del CIITEC y su posgrado. De igual manera fueron consultados y examinados otros documentos normativos del IPN, como su Ley Orgánica, manuales, reglamentos, etc, así como el manual de organización del CIITEC e información proporcionada por el Departamento de Posgrado.

El Posgrado en Tecnología Avanzada (PTA) del CIITEC en sus inicios se vinculó con el Centro de Investigación Aplicada y Tecnología Avanzada (CICATA, unidad Legaria), durante el 2005 y 2006. A partir del primer semestre del 2007, el CIITEC logró el registro de su programa y comienzó a graduar a sus alumnos. Es por esta razón que el análisis inicia en ese semestre. Los plazos manifestados ante Conacyt para la terminación de estudios, incluyendo la tesis y el examen de grado, es de un plazo no mayor de 24 meses para el programa de MTA y 48 meses para el programa de DTA. Como primer paso se examinó la eficiencia terminal del programa de la MTA por semestre (tabla I).

Tabla I. Eficiencia terminal por semestre del programa de la MTA

\begin{tabular}{cccccc}
\hline Semestre & Inscritos & Activos & Graduados & Bajas & $\begin{array}{c}\text { Eficiencia } \\
\text { Terminal \% }\end{array}$ \\
\hline A07 & 4 & 0 & 1 & 3 & 25 \\
B07 & 22 & 0 & 12 & 10 & 54 \\
A08 & 2 & 0 & 2 & 0 & 100 \\
B08 & 4 & 0 & 2 & 2 & 50 \\
A09 & 2 & 0 & 2 & 0 & 100 \\
B09 & 9 & 0 & 7 & 2 & 77 \\
A10 & 7 & 0 & 5 & 2 & 71 \\
B10 & 6 & 0 & 4 & 2 & 66 \\
A11 & 15 & 0 & 14 & 1 & 93 \\
B11 & 7 & 0 & 7 & 0 & 100 \\
A12 & 3 & 0 & 2 & 1 & 66 \\
B12 & 9 & 0 & 8 & 1 & 88 \\
A13 & 7 & 0 & 7 & 0 & 100 \\
B13 & 13 & 0 & 11 & 2 & 84 \\
A14 & 16 & 6 & 9 & 1 & - \\
B14 & 8 & 8 & 0 & 0 & - \\
A15 & 11 & 11 & 0 & 0 & - \\
B15 & 6 & 6 & 0 & 0 & - \\
A16 & 15 & 15 & 0 & 0 & - \\
\hline Totales & 166 & 46 & 93 & 27 & \\
\hline
\end{tabular}

Fuente: Elaboración propia con información del Departamento de Posgrado del CIITEC.

Para determinar la eficiencia terminal de la MTA se tomó en consideración el total de alumnos inscritos, la cantidad de alumnos graduados y el total de alumnos dados de baja por semestre. Los porcentajes muestran que el semestre con menor eficiencia terminal fue el A07 con el 25\%. Los demás semestres expresan la eficiencia terminal por encima del 50\%, siendo los mejores semestres el A08, el A09, el B11 y el A13. Del semestre A07 al semestre B13 se contabilizaron 176 alumnos inscritos, de los cuales 46 continúan activos, 93 se graduaron, y 27 fueron dados de baja. Del semestre A14 en adelante no se obtuvo el porcentaje de eficiencia porque aún hay alumnos activos.

En el programa del DTA se contabilizaron 53 alumnos inscritos de enero 2007 a enero 2016. De los cuales 20 continúan activos, 14 causaron baja y 19 se graduaron. Es necesario mencionar que en los semestres A08, A09 y A10 no hubo alumnos inscritos en este programa. Referente a la eficiencia terminal, en los semestres B07, B09, B10 y B12 está por debajo del 50\%, y en los demás está por encima de este porcentaje; la tabla II muestran estas estadísticas. 
Tabla II. Eficiencia terminal por semestre del programa de DTA

\begin{tabular}{cccccc}
\hline Semestre & Inscritos & Activos & Graduados & Bajas & $\begin{array}{c}\text { Eficiencia } \\
\text { Terminal \% }\end{array}$ \\
\hline A07 & 7 & 0 & 4 & 3 & 57 \\
B07 & 3 & 0 & 1 & 2 & 33 \\
B08 & 3 & 0 & 2 & 1 & 66 \\
B09 & 1 & 0 & 0 & 1 & 0 \\
B10 & 3 & 0 & 1 & 2 & 33 \\
A11 & 2 & 0 & 2 & 0 & 100 \\
B11 & 3 & 0 & 3 & 0 & 100 \\
A12 & 4 & 1 & 2 & 1 & 50 \\
B12 & 5 & 3 & 2 & 0 & - \\
A13 & 6 & 2 & 2 & 2 & - \\
B13 & 3 & 2 & 0 & 1 & - \\
A14 & 3 & 2 & 0 & 1 & - \\
B14 & 2 & 2 & 0 & 0 & - \\
A15 & 1 & 1 & 0 & 0 & - \\
B15 & 3 & 3 & 0 & 0 & - \\
A16 & 4 & 4 & 0 & 0 & - \\
\hline Totales & 53 & 20 & 19 & 14 & \\
\hline
\end{tabular}

Fuente: Elaboración propia con información del Departamento de Posgrado del CIITEC.

El segundo paso fue determinar cómo han influido los profesores en estas estadísticas. Cuál ha sido su papel en la eficiencia terminal de los alumnos. Determinar la eficiencia de los profesores al momento de graduar alumnos si ellos han sido un factor que haya influido en la eficacia terminal de los programas de la MTA y del DTA.

Al momento de hacer la caracterización del núcleo académico básico de profesores del plantel se encontraron los siguientes datos: en total son 16 profesores, 4 mujeres y 12 hombres; 15 tienen grado de doctor y uno de maestro en Ciencias. Realizaron sus estudios de doctorado en diferentes países: 6 en Francia, 3 en México, 2 en Estados Unidos, 2 en Inglaterra, uno en Canadá y uno en Japón. 14 pertenecen al Sistema Nacional de Investigadores (SNI): 4 son nivel dos y 10 son nivel uno. Para completar la información y obtener mayor certeza en las estadísticas, se amplió el estudio a otros 6 profesores que no pertenecen al núcleo académico básico, y se verificó su productividad de alumnos graduados. Con el fin de mantener en el anonimato el nombre de los profesores, se procedió a asignar códigos, denominándolos "docente" y asignándoles un número. Posteriormente se dispuso la información de acuerdo a la eficiencia terminal, del porcentaje mayor al menor.

La tabla III identifica la eficiencia terminal por profesor del programa de la MTA. La tabla contempla información de enero 2007 a febrero 2016. Se cuantificaron 120 alumnos, de ellos 31 permanecen activos, es decir, aún están cursando la maestría; 93 se graduaron, y 27 fueron dados de baja. Se analizó la eficiencia terminal de 22 profesores. Únicamente uno presenta baja eficiencia terminal con 44\%, 10 de ellos están entre el $60 \%$ y $86 \%$ y 11 tienen el $100 \%$.

Cabe destacar que faltan 15 alumnos del semestre A16 en activo, de esa manera sumarían 46, pero no se consideraron en la tabla III porque aún no se les asignaba director de tesis. 
Tabla III. Eficiencia terminal por profesor del programa de MTA

\begin{tabular}{|c|c|c|c|c|}
\hline & Activos & Bajas & Graduados & $\begin{array}{c}\text { Eficiencia } \\
\text { Terminal \% }\end{array}$ \\
\hline Docente 1 & 0 & 0 & 1 & 100 \\
\hline Docente 2 & 0 & 0 & 1 & 100 \\
\hline Docente 3 & 0 & 0 & 1 & 100 \\
\hline Docente 4 & 4 & 0 & 2.5 & 100 \\
\hline Docente 5 & 5 & 0 & 4.5 & 100 \\
\hline Docente 6 & 3 & 0 & 0 & 100 \\
\hline Docente 7 & 2 & 0 & 5 & 100 \\
\hline Docente 8 & 1.5 & 0 & 2 & 100 \\
\hline Docente 9 & 2 & 0 & 4 & 100 \\
\hline Docente 10 & 2 & 0 & 7 & 100 \\
\hline Docente 11 & 2 & 0 & 3 & 100 \\
\hline Docente 12 & 2 & 1 & 6.5 & 86 \\
\hline Docente 13 & 1 & 1 & 6 & 85 \\
\hline Docente 14 & 2 & 1 & 5.5 & 84 \\
\hline Docente 15 & 1 & 1.5 & 7 & 82 \\
\hline Docente 16 & 2 & 2 & 5 & 71 \\
\hline Docente 17 & 2 & 2 & 4.5 & 69 \\
\hline Docente 18 & 2 & 3.5 & 7.5 & 68 \\
\hline Docente 19 & 0 & 1 & 2 & 66 \\
\hline Docente 20 & 1 & 5 & 8 & 61 \\
\hline Docente 21 & 1 & 4 & 6 & 60 \\
\hline Docente 22 & 0 & 5 & 4 & 44 \\
\hline Totales & 31 & 27 & 93 & \\
\hline
\end{tabular}

Fuente: Elaboración propia con información del Departamento de Posgrado del cIITEC.

Por lo que respecta a la eficiencia terminal de los profesores en el programa del DTA, se aplicó el mismo procedimiento. Así, se encontró la eficiencia terminal de 14 docentes, de los cuales 4 tienen una eficiencia terminal del 100\%, 2 del $68 \%$ y $66 \%$, respectivamente, y 8 menor del 50\%. El promedio general de eficiencia terminal es de $55 \%$ en este programa. La tabla IV presenta la descripción de estos porcentajes.

Tabla IV. Eficiencia terminal por profesor del programa de DTA

\begin{tabular}{lcccc}
\hline & Activos & Bajas & Graduados & $\begin{array}{c}\text { Eficiencia } \\
\text { Terminal \% }\end{array}$ \\
\hline Docente 1 & 0 & 0 & 2 & 100 \\
Docente 2 & 1 & 0 & 1.5 & 100 \\
Docente 3 & 3 & 0 & 3.5 & 100 \\
Docente 4 & 3 & 0 & 0.5 & 100 \\
Docente 5 & 1 & 0 & 0.5 & 100 \\
Docente 6 & 1.5 & 2.5 & 5.5 & 68 \\
Docente 7 & 3 & 1 & 2 & 66 \\
Docente 8 & 0.5 & 1.5 & 1 & 40 \\
Docente 9 & 1 & 2 & 1 & 33 \\
Docente 10 & 0 & 2 & 1 & 33 \\
Docente 11 & 1 & 2 & 0.5 & 20 \\
Docente 12 & 1 & 1 & 0 & 0 \\
Docente 13 & 1 & 1 & 0 & 0 \\
\hline Docente 14 & 0 & 1 & 0 & 54 \\
\hline Totales & 12 & 14 & 19 & 0 \\
\hline
\end{tabular}

Fuente: Elaboración propia con información del Departamento de Posgrado del CIITEC. 
Para igualar la cifra de cincuenta y tres alumnos inscritos referidos en la tabla II, es necesario no perder de vista la siguiente información. Sumando los alumnos activos, los graduados y las bajas son cuarenta y cinco alumnos. Faltan por contabilizar ocho alumnos más: uno inscrito en el A15, tres inscritos en el B15 y cuatro en el A16. No están contabilizados porque al momento de hacer las estadísticas aún no se les asigno un profesor como director de tesis.

El tercer paso fue determinar los motivos por los cuales los alumnos fueron dados de baja. Referente a los veintisiete alumnos inscritos en la MTA que fueron dados de baja, el análisis presentó los siguientes resultados: 16 por solicitud propia, de los cuales tres casos fueron por enfermedad y 13 por cambio de programa. Cuatro por abandonar sus estudios sin la autorización correspondiente; y siete por exceder los plazos definidos en el programa. Respecto a los catorce casos de baja de alumnos inscritos en el DTA, la investigación indica que siete de ellos fueron por exceder los plazos definidos en el programa. Cinco abandonaron sus estudios sin la autorización correspondiente; y dos fueron por solicitud propia.

Como se puede ver en los párrafos anteriores solo se observan tres tipos de motivos por los cuales los alumnos son dados de baja, dos de ellos enfocados principalmente al alumno, a solicitud propia y por abandonar sus estudios sin la autorización correspondiente, estos podrían ser atribuibles al alumno y no estar directamente relacionados con el profesor. Donde se observa una posible interrelación entre el alumno y el profesor es al momento de exceder los plazos definidos por los programas para la obtención del diploma o grado correspondiente.

\section{Discusión y conclusiones}

Una característica muy específica de los posgrados en el IPN es el trabajo colegiado que se efectúa en ellos, por medio de un colegio académico constituido por los profesores de los centros de investigación y secciones de Estudios de Posgrado de las Escuelas del IPN. En este sentido, el colegio académico es la máxima autoridad académica de carácter consultivo para la organización y desarrollo de los estudios de posgrado de las unidades académicas del IPN (IPN, 2006), lo que se traduce en una ventaja fundamental en el desarrollo y consolidación de los posgrados.

Por otra parte, una inconsistencia que el estudio evidenció son los plazos para la conclusión de los programas de la MTA. El plazo manifestado ante Conacyt para la terminación de estudios, incluyendo la tesis y el examen de grado, es de 24 meses. Mientras que el Artículo 45 del Reglamento de Estudios de Posgrado del IPN establece que para la terminación de estudios de maestría, incluyendo la tesis y el examen de grado, es de un plazo no mayor de 30 meses (IPN, 2006). De acuerdo con el estudio realizado, el $50 \%$ de los alumnos en el programa del DTA y el $26 \%$ del programa de la MTA exceden los plazos definidos, por lo que se les da de baja, afectando la eficiencia terminal del posgrado.

La eficaz selección de aspirantes en los programas de posgrado del CIITEC podría conllevar una mejor eficiencia terminal. Este supuesto teórico presume que se debe ser más selectivo; de igual manera, se debe dar respuesta a interrogantes de orden cualitativo en los aspirantes, características difíciles de observar y medir cuantitativamente, pero que sin duda los profesores colegiados de mayor experiencia detectarían en una entrevista abierta con los solicitantes. Cabe hacer mención que esta figura de selección en el ingreso al posgrado del CIITEC ya existe, pero no siempre se encuentran en las entrevistas los profesores colegiados de mayor experiencia, por lo cual es una variable importante a considerar.

Como es conocido, la selección de los aspirantes conlleva a cumplir los requisitos normativos y académicos necesarios para su ingreso, evidentemente visibles en la documentación que entregan. La presentación de su currículo ofrece en retrospectiva la información necesaria de su trayectoria académica y laboral. La presentación de un plan de vida del aspirante podría complementar el primero y responder las siguientes interrogantes: ¿Qué factores en el aspirante pueden asegurar que concluya el programa de posgrado hasta acreditar su examen de grado? ¿Qué elementos podrían medir su deseo de continuar estudiando? ¿Qué circunstancias lo motivan a seguir preparándose académicamente? ¿Es por necesidad o lo hace por convicción? ¿Su entorno familiar es propicio? ¿Cuáles son sus expectativas de vida, de crecimiento profesional y de desarrollo personal? ¿Se pueden advertir su disponibilidad y dedicación para 
concluir sus estudios de posgrado? Una estrategia para constatar esta información es instrumentar cursos propedéuticos en los programas de posgrado del CIITEC, con ellos se detectarían debilidades cognitivas, deficiencias en su formación profesional, fortalezas académicas, capacidad inventiva, curiosidad en la investigación y su interés científico en tópicos específicos. El conocimiento de esta información coadyuvaría a fortalecer la certidumbre sobre la eficiencia terminal.

La responsabilidad de la eficiencia terminal en los estudios de posgrado del CIITEC recae en el alumno, en su profesor (director de tesis) y en el propio Centro. Se trata de una responsabilidad compartida. Es una relación simbiótica donde cada quien tiene el compromiso de alcanzar la calidad y la excelencia del Posgrado.

\section{Referencias}

Álvarez, I., Chávez, F., Montiel, J., Topete, C. y Maldonado, N. (2015). Aventuras científicas y tecnológicas de académicos mexicanos. Experiencia de cuatro décadas 1960-2000. México: Limusa.

Álvarez, M., Gómez, E. y Morfín, M. (2012). Efecto de la beca Conacyt en la eficiencia terminal en el posgrado. Revista Electrónica de Investigación Educativa, 14(1), 153-163. Recuperado de https://redie.uabc.mx/redie/article/view/304

Bonilla, A. y Peinado, J. (2014). Semblanza del Centro de Investigación e Innovación Tecnológica del IPN. Hechos que le dieron origen y su evolución. México: Taller Abierto.

Bustos, E., Cerecedo, M. T. y García, M. (2016). Modelo de gestión de conocimiento para el desarrollo del posgrado. Revista Electrónica de Investigación Educativa, 18(1), 128-139. Recuperdo de http://redie.uabc.mx/redie/article/view/579

Cardoso, E. y Cerecedo, M. (2011). Propuesta de indicadores para evaluar la calidad de un programa de posgrado en Educación. Revista Electrónica de Investigación Educativa, 13(2), 68-82. Recuperado de https://redie.uabc.mx/redie/article/view/284

Centro de Investigación e Innovación Tecnológica (2014). Estudio de pertinencia del Posgrado en Tecnología Avanzada del cIITEC. Recuperado de http://www.ciitec.ipn.mx/intranet ciitec/pages/m2e.html

Consejo Nacional de Ciencia y Tecnología (2016). Programa Nacional de Posgrados de Calidad. Recuperado de http://www.Conacyt.mx/index.php/becas-y-posgrados/programa-nacional-de-posgrados-de-calidad

Fadhl, F. y Sofian, S. (2015). A review of balanced scorecard framework in higher education institution (HEIs). International Review of Management and Marketing, 5(1), 26-35.

Hernández, D., Vargas, A., Almuiñas, J. y García, J. (2015). Los indicadores actuales de la eficiencia académica: necesidad de su perfeccionamiento. Pedagogía Universitaria, 20(3), 53-62. Recuperado de http://cvi.mes.edu.cu/peduniv/index.php/peduniv/article/view/690

Instituto Politécnico Nacional (2006). Reglamento de Estudios de Posgrado del IPN. Recuperado de http://www.aplicaciones.abogadogeneral.ipn.mx/reglamentos/rato posgrado.pdf

López A., Albíter A. y Ramírez L. (2008). Eficiencia terminal en la educación superior, la necesidad de un nuevo paradigma. Revista de la Educación Superior, 37(2), 135-151. Recuperado de

http://publicaciones.anuies.mx/pdfs/revista/Revista146 S5A1ES.pdf

Olivares, E. (27 de mayo de 2016). Piden alumnos de posgrado más lugares en maestrías y doctorados en la UNAM. La Jornada, p.38. Recuperado de http://www.jornada.unam.mx/2016/05/27/sociedad/038n2soc 
Ortiz, F. (2011). Diccionario de metodología de la investigación científica. México: Limusa.

Peinado, J. (2009). Propuesta de un programa de capacitación para el personal de apoyo y asistencia a la educación del IPN: estudio de caso Centro de Investigación e Innovación Tecnológica. Tesis de Maestría. Escuela Superior de Comercio y Administración, Instituto Politécnico Nacional, México. Recuperado de http://tesis.ipn.mx/xmlui/bitstream/handle/123456789/5645/PROPUESTAPROGRAMA.pdf?sequence=1\&i sAllowed=y

Peinado, J. (2014). El capital intelectual en los centros de investigación del IPN. Propuesta de un modelo de gestión para el Centro de Investigación e Innovación Tecnológica (CIITEC). Tesis Doctoral, Escuela Superior de Comercio y Administración, Instituto Politécnico Nacional, México.

Peinado, J., Cerecedo, M. y Jaramillo, D. (2016). El balanced scorecard, una herramienta de planeación estratégica en el Centro de Investigación e Innovación Tecnológica del Instituto Politécnico Nacional. Investigación Interdisciplinaria, 2(1), 32-50. Recuperado de http://www.ruii.ipn.mx/index.php/RUIl/article/view/19

Secretaría de Investigación y Posgrado. (2015). Propuesta de indicadores de desempeño de investigación y posgrado 2015. México: IPN.

Yin, R. (2014). Case study research. Design and methods (5a. ed.). Los Ángeles, CA: Sage. 